P173 (continued)

for entrees and lower average diameter (4.92, vs. 5.66 inches), $\mathrm{p}=03$ for baked goods. In our third study we counted the number of shopping bags held by diners at a mall food court and estimated the calories in their meals, consulting objective dietary information when available. There was a significant effect of number of packages carried on calories such that those carrying a greater number of packages ordered more calories of food at the food court: $\mathrm{F}(1,176)=5.47, \mathrm{p}=.02$.

Conclusions and Implications: The studies demonstrate that higher burden leads people to see food quantities as smaller and consume more caloric foods. This suggests consumers may want to avoid reliance on food assessment while burdened.

Funding: Cornell University

\section{P174 Chewing Gum Increases Healthy Restaurant Choices}

AnerTal,PhD, at425@cornell.edu, Cornell University, 38

Meadowlard Drive Lower, Ithaca, NY 14850;

B. Wansink, PhD; S. Miquel-Kergoat, PhD, Wrigley, A

subsidiary of Mars, Inc

Objective: Chewing gum has been shown to reduce snacking (Hetherington and Boyland 2007) because it reduces appetite and suppresses cravings for high-calorie snacks (Hetherington and Regan 2011). However, the question remains whether gum not only reduces consumption of unhealthy snacks but can also guide consumers to opt for healthier options over unhealthy options when choosing food for a meal. This research aimed to examine whether gum can influence the healthiness of one's choices within a meal, such as when ordering at a restaurant, and how immediate this influence might be.

Study Design, Setting, Participants, and Intervention: Participants $(\mathrm{N}=59)$ were randomly assigned to either a gum-chewing group or a non gumchewing group. They were then instructed to pick options out of an on-line restaurant menu devised for the study. Participants were instructed to choose one each of a main dish, side dish, dessert, and drink. For each category, we offered two lower-calorie items, and two higher-calorie items.

Outcome, Measures and Analysis: We counted the number of higher and lower calorie options chosen, and compared these between conditions via t-tests.

Results: Participants who chewed gum picked a higher number of low calorie items (3.38 vs. 2.51 items) and a lower number of high calorie items (.96 vs. 1.6 items). The effects were significant. The effects were significant at a .02 and .02 level respectively.

Conclusions and Implications: Chewing gum while making restaurant choices seems to decrease the percentage of unhealthy choices in a restaurant. This occurs even with a relatively brief period of gum chewing.

Funding: Wrigley Science Advisory Council
P175 Empty Bags, Fuller Stomachs: How Empty Packages Give the Illusion of Fullness

AnerTal,PhD, at425@cornell.edu, Cornell University, 38 Meadowlard Drive Lower, Ithaca, NY 14850;

B. Wansink, PhD

Objective: Leftovers from one's meal serve as an external cue to how much a person has eaten, and consequently to fullness (Wansink 2006). The current study aimed to examine whether empty bags that are left on a table unrelatedly to how much a person has eaten are also taken as an external cue to fullness, leading people to report a higher level of fullness.

Study Design, Setting, Participants, and Intervention: Participants $(\mathrm{N}=63)$ completed the study in exchange for payment. They were randomly assigned to either a small or large bags condition, with the table containing 5 small (1.88 ounces) or 5 large (10 ounces) empty bags respectively. Participants completed the questionnaire at the table containing the bags. The presence of the empty bags was justified by bowls of chips given to participants to eat after completion of satiety measure at the table.

Outcome, Measures and Analysis: Satiety was measured with three 9-point scales measuring hunger, fullness, and desire to eat. We analyzed the effect of bag sizes on satiety using a t-test.

Results: Participants facing large bags reported a higher level of satiety (4.64) than did participants facing small bags (3.8). The difference was significant at a .01 level: $\mathrm{t}(62)=-2.69$.

Conclusions and Implications: It appears that having larger empty bags signaled to participants that they had eaten more, leading them to report a higher level of satiety. Larger bags may be useful for inducing a false sense of satiety.

Funding: Cornell University

\section{P176 Groceries and Gum: Chewing Gum Influences Grocery Store Shopping}

Aner Tal,PhD, at425@cornell.edu, Cornell University, 110 Warren Hall, Ithaca, NY 14850; B. Wansink, PhD;

S. Miquel-Kergoat, PhD, Wrigley, A subsidiary of Mars, Inc

Objective: Impulse shopping in grocery stores - particularly when hungry - has been shown to encourage purchasing an increased percentage of less healthy food, such as potato chips and other ready-to-eat snacks. If one's craving of these foods could be interrupted or otherwise reduced, it could result in healthier shopping behavior.

Study Design, Setting, Participants, and Intervention: A lab study and a grocery store field study were conducted. In the lab study, 59 undergraduates were asked to shop at a simulated online grocery store. Half had been given sugarless chewing gum to chew and half were given nothing. A followup was conducted in the field at a small grocery store with 289 adults. 